\title{
ANALISIS TURUNAN DAN INTEGRAL FRAKSIONAL FUNGSI PANGKAT TIGA DAN FUNGSI EKSPONENSIAL
}

\author{
Muhamad Deni Johansyah ${ }^{1}$, Julita Nahar ${ }^{2}$, Farid H Badruzzaman ${ }^{3}$ \\ ${ }^{1,2}$ Departemen Matematika, FMIPA, Universitas Padjadjaran \\ ${ }^{3}$ Program Studi Matematika FMIPA, Universitas Islam Bandung \\ muhamad.deni@unpad.ac.id, julita.nahar@unpad.ac.id,faridhb@unisba.ac.id
}

\begin{abstract}
Abstrak. Ide dari konsep turunan fraksional adalah bagaimana menentukan turunan yang berorde suatu bilangan pecahan (fraksional) yaitu bilangan rasional atau bahkan bilangan real. Dalam makalah ini dikaji tentang definisi, teorema, dan karakteristik serta contoh turunan fraksional pada fungsi pangkat tiga dan fungsi eksponen yang dikembangkan oleh matematikawan Riemann dan Liouville. Metode penelitian yang ditempuh adalah melalui studi literatur primer, yaitu dengan mengembangkan teori yang telah dikerjakan peneliti terdahulu, sehingga metode tersebut dapat diterapkan untuk penyelesain masalah yang dapat diaplikasikan dan dikembangkan secara lebih luas. Hasil dari makalah ini berupa analisis dari turunan fraksional fungsi pangkat sederhana dan fungsi eksponensial.
\end{abstract}

Keywords: turunan fraksional, fungsi pangkat tiga, fungsi eksponen

\begin{abstract}
The idea of the fractional derivative concept is how to determine the derivation with fractional order, that is a rational number or even a real number. In this paper, we examine the definitions, theorems, and the characteristics as well as fractional derivatives example of the cubic and exponential functions in which developed by mathematician Riemann and Liouville. This research is done by primary literature review, that is by developing the theory that has been done by the previous researcher, so that the method of problem solving can be applied and developed widely. The results of this paper are the analysis of fractional derivatives from simple-order and exponential functions.
\end{abstract}

Keywords: fractional derivatives, cubic functions, exponential functions

\section{Pendahuluan}

Merujuk pada [1], [3] dan [9], turunan fraksional merupakan bagian dari Ilmu Matematika yang kajian teori dan aplikasinya sangatlah luas, termasuk dalam bidang teknik dan industri maupun Ekonomi. Dalam makalah ini akan dianalisis turunan yang berorde fraksional untuk $\alpha=\frac{3}{2}$ pada fungsi pangkat tiga yang berbentuk $\mathrm{f}(\mathrm{x})=a_{1} x^{m_{1}}+a_{2} x^{m_{2}}+a_{3} x^{m_{3}}$ dan fungsi eksponen yang berbentuk $\mathrm{f}(\mathrm{x})=e^{a x}$.

Dalam Kimeu [6] kalkulus fraksional memberikan jawaban atas pertanyaan apakah berlaku sama operasi turunan bilangan bulat berorde $\alpha$ dengan $\alpha$ bukan bilangan bulat. Pada tahun 1819, Lacroix menjadi matematikawan pertama yang memunculkan sebuah paper tentang turunan fraksional. Dimulai dari $y=\mathrm{x}^{\mathrm{m}}$, dengan $\mathrm{m}$ adalah bilangan bulat positif, Lacroix menemukan turunan berorde $\alpha$ sebagai berikut.

$$
\frac{d^{\alpha} y}{d x^{\alpha}}=\frac{m !}{(m-\alpha) !} x^{m-\alpha}, m \geq \alpha
$$

dan dinyatakan dengan menggunakan simbol Legendre $\Gamma$, untuk faktorial umum, Lacroix sebagai berikut

$$
\frac{d^{\alpha} y}{d x^{\alpha}}=\frac{\Gamma(m+1)}{\Gamma(m-\alpha+1)} x^{m-\alpha}
$$


dengan $m=1$ dan $\alpha=\frac{1}{2}$, diperoleh:

$$
\frac{d^{\frac{1}{2}} y}{d x^{\frac{1}{2}}}=\frac{2 \sqrt{x}}{\sqrt{\pi}}
$$

Menurut Podlubny [9], integral dan derivatif fraksional adalah suatu integral dan derivatif dengan orde fraksional (sebarang).Terdapat beberapa pendekatan untuk menotasikan derivatif berorde fraksional antara lain yaitu Riemann-Liouville, Caputo, dan Grunwald-Letnikov. Dalam makalah ini akan dibahas hanya derivatif dan integral fraksional Riemann-Liouville dan Caputo. Definisi Integral fraksional Riemann-Liouville dan definisi dari turunan fraksional telah ada semenjak dua abad terakhir. Merujuk pada [1], [2], [4], [5], dan [7], beberapa definisi yang berkaitan dengan turunan fraksional diantaranya integral fraksional atau turunan fraksional negatif yang dikemukakan oleh Riemann-Liouville yang akan disajikan pada bagian 2. Pada bagian 3 dibahas tentang teorema Integral fraksional dengan pembuktiannya menggunakan definisi 2.1, sedangkan turunan fraksional pembuktiannya menggunakan definisi 2.2. Pada bagian 3 dibahas pula contoh penggunaan definisi dan teorema dari integral dan turunan fraksional. Tujuan dari penelitian adalah untuk mengkaji dan mengetahui definisi, karakteristik dan sifat integral dan turunan fraksional fungsi pangkat tiga dan fungsi eksponensial, sehingga diharapkan dapat berkontribusi secara mendasar pada bidang ilmu Matematika dengan penekanan pada gagasan yang sangat fundamental.

\section{Integral dan Turunan Fraksional}

\subsection{Integral Fraksional}

Sebelum mengkaji tentang integral dan turunan fraksional, akan didefinisikan terlebih dahulu tentang fungsi gamma dan fungsi beta. Menurut Podlubny [9], definisi dari fungsi Gamma adalah

$$
\Gamma(m)=\int_{0}^{\infty} e^{-t} t^{m-1} d t, \quad m \in R^{+}
$$

Fungsi Gamma mempunyai sifat dasar yaitu

$$
\Gamma(m+1)=m \Gamma(m), m \in R^{+}
$$

Dengan menggunakan persamaan (1), fungsi Gamma untuk suatu bilangan bulat positif $m$ dapat dituliskan sebagai

$$
\Gamma(m)=(m-1) !, m \in R^{+}
$$

Berdasarkan persamaan (2) dapat dilihat bahwa fungsi Gamma merupakan perluasan dari fungsi faktorial. Meskipun fungsi Gamma didefinisikan untuk $m>0$, dimungkinkan juga untuk mengembangkan definisi fungsi Gamma untuk semua bilangan riil negatif $m$ yaitu

$$
\Gamma(\mathrm{m})=\frac{1}{\mathrm{~m}} \Gamma(\mathrm{m}+1)
$$

Sedangkan fungsi beta didefinisikan sebagai 


$$
\beta(m, n)=\int_{0}^{1} t^{m-1}(1-t)^{n-1} d t
$$

Konvergen untuk, $n>0$. Adapun hubungan antara fungsi beta dengan fungsi gamma adalah sebagai berikut

$$
\beta(m, n)=\frac{\Gamma(m) \Gamma(n)}{\Gamma(m+n)}
$$

Definisi 2.1 :

Misalkan $\alpha$ bilangan real, integral fraksional orde $\alpha$ dari fungsi $f(x)$ adalah

$$
\begin{aligned}
J^{\alpha} f(x) & =D^{-\alpha} f(x) \\
& =\frac{1}{\Gamma(\alpha)} \int_{0}^{x}(x-t)^{\alpha-1} f(t) d t
\end{aligned}
$$

dengan $\alpha>0$

Sedangkan untuk $\alpha \geq 0$, dan $\beta \geq 0$, integral fraksional yang dikemukakan Riemann-Liouville memiliki sifat sebagai berikut :

1. $J^{\alpha} J^{\beta} f(x)=J^{\alpha+\beta} f(x)$.

2. $J^{\alpha} J^{\beta} f(x)=J^{\beta} J^{\alpha} f(x)$.

Definisi 2.1 mempunyai arti bahwa notasi integral fraksional berorde $\alpha$ dengan batas bawah $x_{0}=$ 0 dari fungsi $\mathrm{f}(\mathrm{x})$ ditulis sebagai $J^{\alpha} f(x)$. Integral fraksional berorde $\alpha$ adalah anti turunan fraksional berorde (- $\alpha)$ untuk fungsi $\mathrm{f}(\mathrm{x})$ yang ditulis sebagai $D^{-\alpha} f(x)$, sehingga berlaku $J^{\alpha} f(x)=D^{-\alpha} f(x)$.

\subsection{Turunan Fraksional}

Defnisi 2.2

Turunan fraksional Riemann-Liouville didefinisikan sebagai

$D_{x_{0}}^{\alpha} f(x)=\frac{d^{n}}{d x^{n}}\left[J^{n-\alpha} f(x)\right]$,

$f(x)=\frac{1}{\Gamma(n-\alpha)} \frac{d^{n}}{d x^{n}}\left[\int_{x_{0}}^{x}(x-t)^{n-\alpha-1} f(t) d t\right]$

dengan $\alpha$ orde sebarang, $n-1 \leq \alpha<n, n \in Z^{+}, x_{0}$ batas bawah, $x_{0}<x, x>0$, dan $D^{\alpha}$ operator derivative fraksional berorde $\alpha$.

Sifat turunan fraksional

1. $D^{\alpha+\beta} f(x)=D^{\alpha} D^{\beta} f(x)$

2. $D^{n}[a f(t)]=a D^{n}[f(t)]$

3. $D^{\alpha}(a f(x)+b g(x))=a D^{\alpha} f(x)+b D^{\alpha} g(x)$

\section{Hasil dan Pembahasan}




\subsection{Fungsi Polinom}

Integral fraksional berorde $\alpha$ dari fungsi polinom sederhana yang berbentuk $\mathrm{f}(\mathrm{x})=x^{m}$, menurut Riemann-Liouville dapat dinyatakan dalam bentuk perkalian fungsi gamma dengan fungsi polinom sebagai berikut:

Persamaan (1.1) untuk $x_{0}=0$ dan $\mathrm{f}(\mathrm{x})=x^{m}$ diperoleh:

$$
J_{0}^{\alpha} \mathrm{f}(\mathrm{x})=J^{\alpha} \mathrm{f}(\mathrm{x})=D^{-\alpha} x^{m}=\frac{1}{\Gamma(\alpha)} \int_{0}^{x}(x-t)^{\alpha-1} t^{m} d t
$$

Sehingga dengan merujuk pada [6] dan [8], kita dapat menghitung $D^{-\alpha} x^{m}$, dimana $\alpha>0, m>-1$. Sebagai berikut

$$
\begin{aligned}
D^{-\alpha} x^{m} & =\frac{1}{\Gamma(\alpha)} \int_{0}^{x}(x-t)^{\alpha-1} t^{m} d t \\
& =\frac{1}{\Gamma(\alpha)} \int_{0}^{x}\left(1-\frac{t}{x}\right)^{\alpha-1} x^{\alpha-1} t^{m} d t \\
& =\frac{1}{\Gamma(\alpha)} \int_{0}^{1}(1-u)^{\alpha-1} x^{\alpha-1}(x u)^{m} x d u \\
& =\frac{1}{\Gamma(\alpha)} x^{m+\alpha} \int_{0}^{1}(1-u)^{\alpha-1}(u)^{m} d u \\
& =\frac{1}{\Gamma(\alpha)} x^{m+\alpha} B(m+1, \alpha) \\
D^{-\alpha} x^{m} & =\frac{\Gamma(m+1)}{\Gamma(m+\alpha+1)} x^{m+\alpha}
\end{aligned}
$$

Sehingga integral fraksional berorde $\alpha$ dari fungsi polinom sederhana yang berbentuk $f(x)=x^{m}$ dapat dinyatakan dalam bentuk perkalian fungsi gamma dengan fungsi polinom yang dapat dinyatakan dalam teorema berikut:

Teorema 2.1:

Integral fraksional berorde $\alpha$ dari fungsi polinom sederhana yang berbentuk

$f(x)=x^{m}$ adalah

$J^{\alpha} x^{m}=\frac{\Gamma(m+1)}{\Gamma(m+\alpha+1)} x^{m+\alpha}$, untuk $\alpha>0, m>-1, x>0$

Kita lihat teorema (2.1) menginformasikan kita, bahwa integral fraksional dari suatu k yang konstan berorde $\alpha$ adalah:

$J^{\alpha} k=J^{\alpha} k \cdot x^{0}=\frac{k \cdot \Gamma(0+1)}{\Gamma(0+\alpha+1)} x^{0+\alpha}=\frac{k}{\Gamma(\alpha+1)} x^{\alpha}$

Selanjutnya akan dibahas Integral fraksional $J^{\alpha} f(Q)$ dari fungsi

$\mathrm{f}(\mathrm{Q})=\mathrm{aQ}^{3}+\mathrm{bQ}^{2}+\mathrm{cQ}$, untuk $\alpha=\frac{3}{2}$ dan $\mathrm{Q}=1$ dengan menggunakan definisi 2.1 sebagai berikut: 
Misalkan $\alpha$ bilangan real, integral fraksional orde $\alpha$ dari fungsi $f(x)$ adalah:

$$
J^{\alpha} f(x)=D^{-\alpha} f(x)=\frac{1}{\Gamma(\alpha)} \int_{0}^{x}(x-t)^{\alpha-1} f(t) d t
$$

dengan $\alpha>0$. Sehingga diperoleh:

$$
\begin{aligned}
& \text { 1) } J^{\frac{3}{2}} b Q^{3}=\frac{a}{\Gamma\left(\frac{3}{2}\right)}{ }_{0} \int^{Q}(Q-t)^{3 / 2-1} t^{3} \mathrm{dt}=\frac{2 b}{\sqrt{\pi}} \int_{0}^{Q}(Q-t)^{1 / 2} \cdot t^{3} \mathrm{dt} \\
& \text { misal : } \mathrm{t}=\mathrm{Qu}, \quad \mathrm{Q}=0 \rightarrow \mathrm{t}=0 \\
& \mathrm{dt}=\mathrm{Qdu}, \quad \mathrm{u}=\mathrm{Q} \rightarrow \mathrm{t}=1 \\
& J^{\frac{3}{2}} b Q^{3}=\frac{2 b}{\sqrt{\pi}} \mathrm{o} \int^{1}(\mathrm{Q}-\mathrm{Qu})^{1 / 2} Q^{3} u^{3} \cdot \mathrm{Qdu}=\frac{2 b}{\sqrt{\pi}}{ }_{0} \int^{1}(\mathrm{Q}(1-\mathrm{u}))^{1 / 2} Q^{4} u^{3} \mathrm{du} \\
& J^{\frac{3}{2}} b Q^{3}=\frac{2 b Q^{\frac{9}{2}}}{\sqrt{\pi}}{ }_{0} \int^{1} u^{3} \cdot(1-\mathrm{u})^{1 / 2} \mathrm{du}=\frac{2 b Q^{\frac{9}{2}}}{\sqrt{\pi}} \beta\left(4, \frac{3}{2}\right)=\frac{2 b Q^{\frac{9}{2}}}{\sqrt{\pi}} \cdot \frac{\Gamma(4) \Gamma\left(\frac{3}{2}\right)}{\Gamma\left(\frac{11}{2}\right)} \\
& =2 b \frac{Q^{\frac{9}{2}}}{\sqrt{\pi}} \frac{3 \sqrt{\pi}}{\frac{9}{2} \cdot \frac{7}{2} \cdot \frac{5}{2} \cdot \frac{3}{2} \cdot \frac{1}{2} \sqrt{\pi}}=\frac{64 b}{315} \sqrt{\frac{Q^{7}}{\pi}}
\end{aligned}
$$

2) $J^{\frac{3}{2}} b Q^{2}=\frac{b}{\Gamma\left(\frac{3}{2}\right)}{ }_{0}{ }^{Q}(Q-t)^{3 / 2-1} t^{2} \mathrm{dt}=\frac{2 b}{\sqrt{\pi}} \int^{Q}(Q-t)^{1 / 2} \cdot t^{2} \mathrm{dt}$

$$
\begin{aligned}
\operatorname{misal}: \mathrm{t} & =\mathrm{Qu}, \quad \mathrm{Q}=0 \rightarrow \mathrm{t}=0 \\
\mathrm{dt} & =\mathrm{Qdu}, \quad \mathrm{u}=\mathrm{Q} \rightarrow \mathrm{t}=1
\end{aligned}
$$$$
J^{\frac{3}{2}} b Q^{2}=\frac{2 b}{\sqrt{\pi}} \int^{1}(\mathrm{Q}-\mathrm{Qu})^{1 / 2} Q^{2} u^{2} \cdot \mathrm{Qdu}=\frac{2 b}{\sqrt{\pi}}{ }_{\mathrm{o}} \int^{1}(\mathrm{Q}(1-\mathrm{u}))^{1 / 2} Q^{3} u^{2} \mathrm{du}
$$$$
J^{\frac{3}{2}} b Q^{2}=\frac{2 b Q^{\frac{7}{2}}}{\sqrt{\pi}} \text { o } \int{ }^{1} u^{2} \cdot(1-\mathrm{u})^{1 / 2} \mathrm{du}=\frac{2 b Q^{\frac{7}{2}}}{\sqrt{\pi}} \beta\left(3, \frac{3}{2}\right)=\frac{2 b Q^{\frac{7}{2}}}{\sqrt{\pi}} \cdot \frac{\Gamma(3) \Gamma\left(\frac{3}{2}\right)}{\Gamma\left(\frac{9}{2}\right)}
$$$$
=2 b \frac{Q^{\frac{7}{2}}}{\sqrt{\pi}} \frac{\sqrt{\pi}}{\frac{7}{2} \cdot \frac{5}{2} \cdot \frac{3}{2} \cdot \frac{1}{2} \sqrt{\pi}}=\frac{32 b}{105} \sqrt{\frac{Q^{7}}{\pi}}
$$

3) $J^{\frac{3}{2}} c Q^{1}=\frac{c}{\Gamma\left(\frac{3}{2}\right)}{ }_{0} \int^{Q}(Q-t)^{\frac{1}{2}-1} \cdot \mathrm{tdt}=\frac{c}{\sqrt{\pi}} \mathrm{o} \int^{x}(Q-t)^{\frac{1}{2}} \cdot \mathrm{tdt}=\frac{8 c}{15} \sqrt{\frac{Q^{5}}{\pi}}$

Dengan demikian dari 1), 2), dan 3) integral fraksional dari

$\mathrm{f}(\mathrm{Q})=\mathrm{aQ}^{3}+\mathrm{bQ}^{2}+\mathrm{cQ}$ Untuk $\alpha=\frac{3}{2}$ adalah

$J^{\frac{3}{2}} \mathrm{f}(\mathrm{Q})=\frac{64 a}{315} \sqrt{\frac{Q^{7}}{\pi}}+\frac{32 b}{105} \sqrt{\frac{Q^{7}}{\pi}}+\frac{8 c}{15} \sqrt{\frac{Q^{5}}{\pi}}$, sedangkan untuk $\mathrm{Q}=1$, diperoleh

$$
J^{\frac{3}{2}} \mathrm{f}(1)=\frac{64 a}{315} \sqrt{\frac{1}{\pi}}+\frac{32 b}{105} \sqrt{\frac{1}{\pi}}+\frac{8 c}{15} \sqrt{\frac{1}{\pi}}=\frac{64 a+96 b+168 c}{315} \frac{\sqrt{\pi}}{\pi}
$$

Fungsi turunan fraksional bisa diartikan dengan menggunakan definisi integrasi fungsi, karena definisi 2.1 mempunyai arti bahwa notasi integral fraksional berorde $\alpha$ dari fungsi $\mathrm{f}(\mathrm{x})$ adalah anti turunan fraksional berorde $(-\alpha)$ untuk fungsi $\mathrm{f}(\mathrm{x})$ yang ditulis sebagai $D^{-\alpha} f(x)$, sehingga berlaku $J^{\alpha} f(x)=D^{-\alpha} f(x)$. Sampai saat ini, asumsi bahwa $\alpha=n-u$, dimana $0<\alpha<1$ dan $\mathrm{n}$ adalah bilangan bulat terkecil yang lebih besar dari $u$. Lalu, fungsi turunan $f(x)$ dari urutan $u$ adalah:

$$
D^{u} f(x)=D^{n}\left[D^{-\alpha} f(x)\right]
$$


Asumsikan kita ingin mencari fungsi turunan fraksional $f(x)=x^{m}$ untuk turunan $\alpha$, dimana $m \geq 0$. Perhatikan apabila kita ingin menggunakan teorema (2.1), kita harus menukar $u$ dan $\alpha$, misalkan $u=n-\alpha$, di mana $0<u<1$. Lalu kita punya $n=1$ dan $u=1-\alpha$, sehingga dengan merujuk pada [6] dan [8], maka diperoleh:

$$
\begin{aligned}
D^{\alpha} f(x) & =D^{1}\left[D^{-(1-\alpha)} f(x)\right] \\
& =D^{1}\left[D^{-(1-\alpha)} x^{m}\right] \\
& =D^{1}\left[\frac{\Gamma(m+1)}{\Gamma((m-\alpha+1)+1)} x^{m-\alpha+1}\right] \\
& =(m-\alpha+1) \frac{\Gamma(m+1)}{(m-\alpha+1) \Gamma(m-\alpha+1)} x^{m-\alpha} \\
& =\frac{\Gamma(m+1)}{\Gamma(m-\alpha+1)} x^{m-\alpha}
\end{aligned}
$$

Sehingga turunan fraksional berorde $\alpha$ dari fungsi polinom sederhana yang berbentuk $f(x)=x^{m}$, dapat dinyatakan dalam bentuk perkalian fungsi gamma dengan fungsi polinom yang dapat dinyatakan dalam teorema berikut:

\section{Teorema 2.2 :}

Turunan fraksional berorde $\alpha$ dari fungsi polinom sederhana yang berbentuk $f(x)=x^{m}$ adalah

$$
D^{\alpha} x^{m}=\frac{\Gamma(m+1)}{\Gamma(m-\alpha+1)} x^{m-\alpha}, \text { untuk } m \geq 0,0<\alpha<1
$$

kemudian dicari turunan fraksional berode $\alpha=\frac{1}{2}$ dari suatu fungsi konstanta $\mathrm{f}(\mathrm{t})=C$, dengan menggunakan teorema 2.2 sebagai berikut:

$$
\begin{aligned}
D_{t}^{\frac{1}{2}}[C]=D_{t}^{\frac{1}{2}}\left[C \cdot t^{0}\right] & =C \frac{\Gamma(0+1)}{\Gamma\left(0-\frac{1}{2}+1\right)} t^{-\frac{1}{2}} \\
& =C \frac{\Gamma(1)}{\Gamma\left(\frac{1}{2}\right)} t^{-\frac{1}{2}}=\frac{C}{\sqrt{\pi t}}
\end{aligned}
$$

Dengan demikian dapat ditarik kesimpulan bahwa turunan fraksional berorde $\alpha$ dari suatu fungsi konstanta tidaklah nol.

Notasi untuk $D_{t}^{\alpha} f(x)$ untuk t di sekitar 0 dapat ditulis sebagai $D^{\alpha} f(x)$. Selanjutnya dalam bagian ini akan dibahas turunan fraksional dari fungsi $f(Q)=a Q^{3}+b Q^{2}+c Q$, dengan menggunakan teorema 2.2, untuk orde $\alpha=3 / 2$ dan $\mathrm{Q}=1$, sebagai berikut:

Turunan fraksional berorde $\alpha$ dari fungsi polinom sederhana yang berbentuk $\mathrm{f}(\mathrm{x})=\mathrm{x}^{\mathrm{m}}$ adalah

$$
D^{\alpha} x^{m}=\frac{\Gamma(m+1)}{\Gamma(m-\alpha+1)} x^{m-\alpha}, \text { untuk } m \geq 0,0<\alpha<1
$$

Sehingga turunan fraksional berorde $\alpha=3 / 2$, untuk $x_{0}=0$ dari $\mathrm{f}(\mathrm{Q})=\mathrm{aQ}^{3}+\mathrm{bQ}^{2}+\mathrm{cQ}+\mathrm{d}$, adalah
1) $D^{\frac{3}{2}} a Q^{3}=a \frac{\Gamma(3+1)}{\Gamma\left(3-\frac{3}{2}+1\right)} Q^{3-\frac{3}{2}}=a \frac{\Gamma(4)}{\Gamma\left(\frac{5}{2}\right)} Q^{\frac{3}{2}}=\frac{6 a}{\frac{3}{2} \cdot \frac{1}{2} \sqrt{\pi}} Q^{\frac{3}{2}}=8 a \sqrt{\frac{Q^{3}}{\pi}}$
2) $D^{\frac{3}{2}} b Q^{2}=b \frac{\Gamma(2+1)}{\Gamma\left(2-\frac{3}{2}+1\right)} Q^{2-\frac{3}{2}}=b \frac{\Gamma(3)}{\Gamma\left(\frac{3}{2}\right)} Q^{\frac{1}{2}}=\frac{2 b}{\frac{1}{2} \sqrt{\pi}} Q^{\frac{1}{2}}=4 b \sqrt{\frac{Q}{\pi}}$ 
3) $D^{\frac{3}{2}} c Q^{1}=c \frac{\Gamma(1+1)}{\Gamma\left(1-\frac{3}{2}+1\right)} Q^{1-\frac{3}{2}}=c \frac{\Gamma(2)}{\Gamma\left(\frac{1}{2}\right)} Q^{-\frac{1}{2}}=\frac{c}{\sqrt{\pi}} Q^{-\frac{1}{2}}=\frac{c}{\sqrt{\pi Q}}$

Dengan demikian dari 1), 2), dan 3) diperoleh turunan fraksional dari $f(Q)=a Q^{3}+b Q^{2}+c Q$, dengan orde

$\alpha=\frac{3}{2}$ adalah $D^{\frac{3}{2}} f(Q)=8 a \sqrt{\frac{Q^{3}}{\pi}}+4 b \sqrt{\frac{Q}{\pi}}+\frac{c}{\sqrt{\pi Q}}$, sedangkan untuk $Q=1$ diperoleh:

$D^{\frac{3}{2}} f(1)=\frac{8 a+4 b+c}{\sqrt{\pi}}$.

Dengan demikian turunan fraksional untuk $\alpha=\frac{3}{2}$ dan $\mathrm{Q}=1$ dari fungsi

$\mathrm{f}(\mathrm{Q})=\mathrm{aQ} \mathrm{Q}^{3}+\mathrm{bQ} \mathrm{Q}^{2}+\mathrm{cQ}$ adalah $D^{\frac{3}{2}} f(1)=\frac{8 a+4 b+c}{\sqrt{\pi}}$.

\subsection{Eksponensial}

Dalam Xuru, 2006 [10], pada kasus fungsi eksponensial yang sederhana memberikan beberapa petunjuk tentang generalisasi turunan, sebagai berikut:

$$
\begin{aligned}
D^{\alpha} e^{k x} & =\lim _{h \rightarrow 0} h^{-\alpha} \sum_{m=0}^{\alpha}(-1)^{m}\left(\begin{array}{c}
\alpha \\
m
\end{array}\right) e^{k(x+(\alpha-m) h)} \\
& =e^{k x} \lim _{h \rightarrow 0} h^{-\alpha} \sum_{m=0}^{\alpha}(-1)^{m}\left(\begin{array}{c}
\alpha \\
m
\end{array}\right)\left(e^{k h}\right)^{\alpha-m} \\
& =e^{k x} \lim _{h \rightarrow 0} h^{-\alpha}\left(e^{k h}-1\right)^{\alpha} \\
& =k^{\alpha} e^{k x}
\end{aligned}
$$

Dengan demikian diperoleh turunan fraksional dari fungsi kompleks dengan bagian real adalah fungsi cosinus dan bagian imajiner fungsi sinus sebagai berikut:

$$
\begin{aligned}
D^{\alpha} \cos (x)+i D^{\alpha} \sin (x) & =D^{\alpha} e^{i x}=i^{\alpha} e^{i x} \\
& =e^{\frac{\alpha \pi i}{2}} e^{i x}=e^{i\left(x+\frac{\alpha \pi}{2}\right)} \\
& =\cos \left(x+\frac{\alpha \pi}{2}\right)+i \sin \left(x+\frac{\alpha \pi}{2}\right)
\end{aligned}
$$

dan

$$
\begin{aligned}
D^{\alpha} \cos (x)-i D^{\alpha} \sin (x) & =D^{\alpha} e^{-i x}=(-i)^{\alpha} e^{-i x} \\
& =e^{\frac{-\alpha \pi i}{2}} e^{-i x}=e^{-i\left(x+\frac{\alpha \pi}{2}\right)} \\
D^{\alpha} \cos (x)-i D^{\alpha} \sin (x) & =\cos \left(x+\frac{\alpha \pi}{2}\right)-i \sin \left(x+\frac{\alpha \pi}{2}\right)
\end{aligned}
$$

Sehingga kita mendapatkan turunan sinus dan cosinus sebagai berikut:

$$
D^{\alpha} \sin (x)=\sin \left(x+\frac{\alpha \pi}{2}\right)
$$

dan

$$
D^{\alpha} \cos (x)=\cos \left(x+\frac{\alpha \pi}{2}\right)
$$


Kita bisa mengharapkan relasi ini untuk turunan sinus dan cosinus untuk dipertahankan dalam generalisasi turunan. Dengan cara menerapkan metode tersebut di atas kita juga bisa menentukan bentuk turunan fraksional dari fungsi cosinus dan fungsi sinus sebagai berikut:

$$
\begin{aligned}
D^{\alpha} \cos (k x)+i D^{\alpha} \sin (k x) & =D^{\alpha} e^{i k x}=(k i)^{\alpha} e^{i k x} \\
& =k^{\alpha} e^{\frac{\alpha \pi i}{2}} e^{i a x}=k^{\alpha} e^{i\left(k x+\frac{\alpha \pi}{2}\right)} \\
& =k^{\alpha} \cos \left(k x+\frac{\alpha \pi}{2}\right)+i k^{\alpha} \sin \left(k x+\frac{\alpha \pi}{2}\right)
\end{aligned}
$$

dan

$$
\begin{aligned}
D^{\alpha} \cos (k x)-i D^{\alpha} \sin (k x) & =D^{\alpha} e^{-i k x}=(-k i)^{\alpha} e^{-i k x} \\
& =k^{\alpha} e^{-\frac{\alpha \pi i}{2}} e^{-i k x}=k^{\alpha} e^{-i\left(k x+\frac{\alpha \pi}{2}\right)} \\
& =k^{\alpha} \cos \left(k x+\frac{\alpha \pi}{2}\right)-i k^{\alpha} \sin \left(k x+\frac{\alpha \pi}{2}\right)
\end{aligned}
$$

Dengan demikian, diperoleh:

$$
D^{\alpha} \sin (k x)=k^{\alpha} \sin \left(k x+\frac{\alpha \pi}{2}\right)
$$

dan

$D^{\alpha} \cos (k x)=k^{\alpha} \cos \left(k x+\frac{\alpha \pi}{2}\right)$

\section{Kesimpulan Dan Saran}

(1) Turunan fraksional adalah bentuk yang lebih umum dari turunan berode bilangan bulat. Berbeda dengan turunan berorde bilangan bulat dimana operasi berpusat pada bilangan bulat, akan tetapi turunan fraksional menganggap setiap bilangan adalah real, dengan $\alpha>0$.

(2) Integral fraksional merupakan alat untuk mencari turunan fraksional fungsi polinom maupun fungsi eksponen, sedangkan turunan fraksional dari fungsi eksponensial dapat digunakan sebagai alat untuk mencari turunan fraksional dari fungsi sinus dan fungsi cosinus.

\section{Ucapan Terima Kasih}

Terima kasih kepada Rektor Universitas Padjadjaran yang telah memberikan bantuan melalui hibah internal RFU (Riset Fundamental UNPAD) tahun 2017.

\section{Referensi}

[1] Adam Loverro, (2004), Fractional Calculus: History, Definitions, and Applications for the Engineer, University of Notre Dame.

[2] Bologna, Mauro, (2015), Short Introduction to Fractional Calculus.Chile: Universidad deTarapaca. Available 20 May 2015.

https://www.researchgate.net/profile/Mauro_Bologna2/publication/268373751_Short_Introduction_to_Fractional_Ca lculus/links/555c7b3508ae91e75e77931f.pdf

[3] E. Rusyaman, H. Gunawan, A.K. Supriatna, dan R.E. Siregar, 2009, A 2-D Interpolation Method That Minimizes An Energy Integral, Presented at IICMA in Yogyakarta.

[4] Indian Electronic These and Dissertations. Chapter 1: Introduction to Fractional Calculus.http://shodhganga.inflibnet.ac.in/bitstream/10603/9663/7/07_chapter\%201.pdf 
[5] Ishteva, Mariya Kamenova, (2005), Properties and Applications of the Caputo Fractional Operator (Thesis). Bulgaria: Department of Mathematics. Universitas Karlsrube (TH) February 2005. http://homepages.vub.ac.be/ mishteva/papers/Ishteva_MScThesis.pdf

[6] Kimeu, Joseph. (2009). Fractional Calculus: Defnitions and Applications (Thesis). Kentucky: The Faculty of the Department of Mathematics. Western Kentucky University. http://digitalcommons.wku.edu/cgi/viewcontent.cgi?article=1115\&context=theses.

[7] Kisela, Thomas, (2008), Fractional Differential Equations and Their Applications (Thesis). Brno, Czech Republic: Faculty of Mechanical Engineering Institute of Mathematics. BRNO University of Technology. https://www.researchgate.net/profile/Tomas_Kisela/publication/249993249_Fractional_Differential_Equations_and_ Their_Applications/links/00b7d51e821756d8fb000000.pdf

[8] McTier, Austin, (2016), Fractional Calculus Fundamentals and Applications in Economic Modeling (Thesis). Georgia: Georgia College and State University. 12 December 2016. https://www.gcsu.edu/sites/files/page-assets/node808/attachments/mctier.pdf

[9] Podlubny, Igor. (1999), Fractional Differential Equations, Technical University of Kosice, Slovakia, Academic Press.

[10] Xuru, 2006, Catatan Pengantar untuk Kalkulus Fraksional. 